\title{
Mammary Tuberculosis in Male
}

\section{Siddharth Pramod Dubhashi, Riddhima Shailesh Dubhashi}

Correspondence: Dr. Siddharth Pramod Dubhashi, All India Institute of Medical Sciences, Nagpur, India.

Email: spdubhashi@gmail.com

\begin{abstract}
Mammary Tuberculosis is rare, with an incidence of $4 \%$ of all breast diseases in TB endemic countries. The clinical presentation can be non-specific and may mimic malignancy of the breast. This is a case report of a middle-aged male patient presenting with an ulcerated lump of the breast, diagnosed as mammary tuberculosis. Breast tuberculosis is extremely rare in males. The clinical presentation is varied and a high index of clinical suspicion is extremely essential for diagnosis with confirmation by cytology and histology. Anti-tubercular treatment (ATT) is the mainstay of treatment, with surgery being required in few indicated cases.
\end{abstract}

Keywords: ATT; Disseminated; Histology; Male breast; Tuberculosis.

DOI: https://doi.org/10.3126/jssn.v23i1.33555

\section{Introduction}

Mammary Tuberculosis is rare, with an incidence of $4 \%$ of all breast diseases in TB endemic countries. ${ }^{1}$ The clinical presentation can be non-specific and may mimic malignancy of the breast. Tuberculosis of breast usually affects young, multiparous, lactating women, but it can also affect males. ${ }^{2}$ It may be primary or secondary as a result of haematogenous spread from axillary lymph nodes or direct extension from lung and mediastinum. ${ }^{3}$ This is a case report of a middle-aged male patient presenting with an ulcerated lump of the breast, diagnosed as mammary tuberculosis.

\section{Case Report}

A 42 years old male patient presented to the surgical OPD with a lump in the right breast involving the upper and lower outer quadrants, extending into the axilla with evidence of skin ulceration (Figure 1). As per history narrated by the patient, the lump had been present for the past four months and the skin involvement was evident for one week. The breast lump was tender, diffuse and irregular, with overlying skin oedema. There was no clinical evidence of axillary lymphadenopathy. There was no history of fever, however, the patient did mention about significant weight loss. The haemogobin value, total leucocyte count and chest X-Ray were normal. Fine Needle Aspiration Cytology (FNAC) reported an acute inflammatory exudate. The discharge from the lesion at the site of ulceration was then subjected to Ziehl-Neelsen staining and culture for acid-fast bacilli,

Journal of Society of Surgeons of Nepal (JSSN) which proved negative for the same. Considering the large size of the lesion and the ulcerated discharging lesion being present for four months, the lump was excised in-toto along with the adherent axillary tissue. The histopathological examination revealed typical granulomatous and Langhans giant cells (Figure 2) with the involvement of four axillary lymph nodes.

Anti-tubercular treatment (ATT) was administered to the patient for six months ( two months of intensive phase with Isoniazid, Rifampicin, Pyrazinamide and Ethambutol and four months with Isoniazid and Rifampicin). The patient has been asymptomatic during a follow-up of two years.

\section{Discussion}

Mammary tuberculosis was first described by Sir Astley Cooper in 1829, who called it "scrofulous swelling of bosom". It may be primary when no demonstrable tuberculous focus exists, or secondary to a lesion elsewhere in the body. ${ }^{5}$ Breast provides an infertile environment for survival and multiplication of tubercle bacilli. The infection may spread via haematogenous, lymphatic routes, contiguous structures, direct inoculation and ductal infection. Communications between the axillary glands and the breast result in secondary involvement of the breast by retrograde lymphatic extension. ${ }^{6}$ In our case, involvement of four axillary lymph nodes was evident in the pathological examination. However, whether the axilla was the primary site of infection or secondary to breast involvement is a point of debate.

JSSN 2020; $23(1)$ 


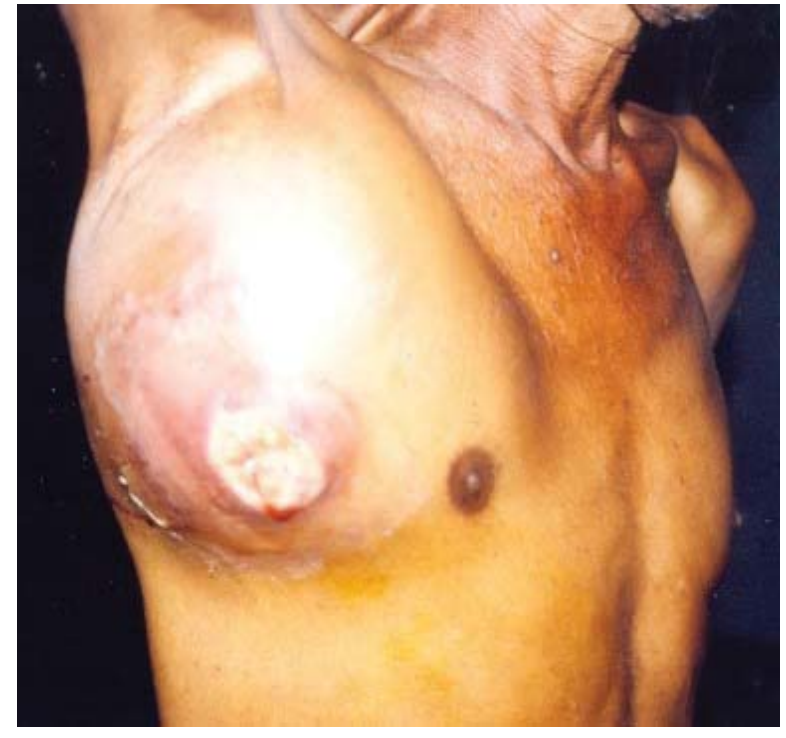

Figure 1. Ulcerated lump involving right breast and axilla

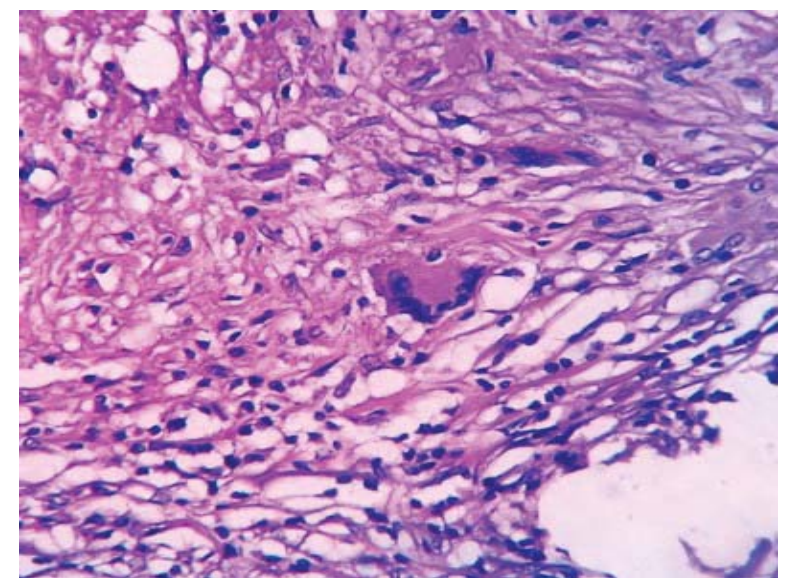

Figure 2. H\&E stained section showing Langhans multinucleated giant cells (40X magnification)

Breast tuberculosis is extremely rare in males, with the first case being reported in 1927. ${ }^{7}$ Few case reports on male breast tuberculosis reveal that the presentation is of a unilateral breast mass with or without ulceration along with axillary lymphadenopathy. ${ }^{8}$ Discharging sinuses and purulent nipple discharge are rare presenting features. ${ }^{6}$ McKeown and Wilkinson ${ }^{5}$ have classified breast tuberculosis into five types- nodular tubercular mastitis, disseminated or confluent tubercular mastitis, sclerosing tubercular mastitis, tuberculous mastitis obliterans and acute miliary tubercular mastitis. The present case fits into the category of disseminated form of tuberculous mastitis, with the overlying skin being stretched with an ulcerated lesion and involvement of axillary lymph nodes. Currently, however, three types are classified- Nodular, disseminated and abscess varieties. ${ }^{6}$
The diagnosis of this condition warrants a high index of clinical suspicion along with pathological and microbiological evidence. Khanna et $\mathrm{al}^{8}$ have mentioned FNAC or histology being $100 \%$ reliable in diagnosing tuberculosis, including patients who had tender nodularity. Bacteriological examination of discharge from the ulcers or sinuses was negative for $\mathrm{M}$. tuberculosis on staining as well as culture in most cases. ${ }^{9}$

Other imaging modalities like mammography or ultrasonography cannot differentiate tuberculous mastitis from malignancy due to non-specific features. Mammography demonstrates a coarse stromal texture with or without an ill-defined breast lesion. Ultrasound shows illdefined hypoechoic masses in nodular and diffuse lesions and increased echogenicity in sclerosing variety. Computed Tomography scan may help in defining the thoracic wall involvement in deeply adhered breast lumps. MRI offers no added advantage in diagnosis, except for utility in assessing the extra-mammary extent of the lesion. ${ }^{6,8,10}$

Treatment comprises of anti-tubercular therapy (ATT) and surgery with specific indications like- poor response to ATT, drainage of cold abscesses, and excision of residual lumps. ${ }^{6,11}$ Our case had a large painful lump with an ulcerative lesion which warranted excision, even for diagnosis. Standard ATT for 6 months usually results in good clinical response. . $^{1,6,11}$

\section{Conclusion}

Breast tuberculosis is rare, and in a male patient is a unique entity. The clinical presentation is varied and a high index of clinical suspicion is extremely essential for diagnosis with confirmation by cytology and histology. Anti-tubercular treatment is the mainstay of treatment, with surgery being required in a few indicated cases.

\section{References}

1. Akcakaya A, Eryilmaz R, Sahin M, Ozkan OV. Tuberculosis of the breast. Breast J. Jan-Feb 2005;11(1):85-6.

2. Jaideep C, Kumar M, Khanna AK. Male breast tuberculosis. Postgrad Med J. 1997 Jul;73(861):428-9.

3. Zandrino F, Monetti F, Gandolfo N. Primary tuberculosis of the breast. A case report. Acta Radiol. 2000 Jan;41(1):61-3.

4. Cooper A. Illustrations of the diseases of the breast. Part I. London: Longman Rees Orme Brown and Green;1829.73p. 
5. McKeown KC, Wilkinson KW. Tuberculous disease of the breast. Br J Surg. 1952 Mar;39(157):420.

6. Tewari M, Shukla HS. Breast tuberculosis: diagnosis, clinical features and management. Indian J Med Res. 2005 Aug;122(2):103-10.

7. Dickinson AM. Mammary tuberculosis: report of a case in the male. Am J Surg 1927 Dec;3(6):595-7.

8. Khanna R, Prasanna GV, Gupta P, Kumar M, Khanna S, Khanna AK. Mammary tuberculosis: report on 52 cases. Postgrad Med J. 2002 Jul;78(921):422-4.

9. Bani-Hani KE, Yaghan RJ, Matalka II, Mazahreh TS. Tuberculous mastitis: a disease not to be forgotten. Int J Tuberc Lung Dis. 2005 Aug;9(8):920-5.

10. Al-Marri MR, Aref E, Omar AJ. Mammographic features of isolated tuberculous mastitis. Saudi Med J. 2005 Apr;26(4):646-50.

11. Sriram KB, Moffatt D, Stapledon R. Tuberculosis infection of the breast mistaken for granulomatous mastitis: a case report. Cases J. 2008 Oct 25;1(1):273. 